\title{
PREVENTION AND TREATMENT OF HAND INFECTIONS
}

\author{
T. G. Lowden, M.A., F.R.C.S. \\ Surgeon, Royal Infirmary, Sunderland
}

Most hand infections cannot be traced to a precise source, though it is likely that in all of them a minor, unnoticed breach of skin surface has allowed entry of pyogenic organisms. Prophylaxis is therefore difficult, but attention to pricks, small cuts or abrasions as soon as they are sustained, simple cleansing or the early application of a protective dressing will help to reduce them. Industrial and household gloves and barrier creams are also useful. The chance of a minor injury giving rise to a hand infection is increased in those whose skin habitually harbours pathogens. Williams and Miles (1949) showed that hand infections after minor injury occur more often in carriers of staphylococcus aureus, and the infecting organism often corresponds with organisms isolated from the neighbouring skin. A correspondence between infecting strain and a strain carried in the nose has also been noted. This should be considered in patients who say that their minor wounds often go septic. Where it is possible, reservoirs of infection should be eliminated.

\section{Bacteriology}

In a small number of cases streptococcus pyogenes is probably primarily responsible. In some staphylococcus aureus is associated with streptococcus pyogenes, and in these the staphylococcus usually determines the clinical character of the infection. The majority are due to staphylococcus aureus. Coliforms are occasionally isolated in pure culture, especially from finger-tip lesions. Coliforms, pseudomonas pyocyaneus, streptococcus pyogenes, and strains of staphylococcus aureus other than the causative strain may appear in lesions with persistent discharge and a protracted course. In clinics and hospitals the tendency for samples of staphylococcus aureus to show antibiotic resistance increases with the time taken for the lesions to heal. Most cases of septic hand are contracted in industry or in the home and the strains encountered there are predominantly penicillin sensitive. But if it is suspected that infection has started in hospital, amongst hospital or medical staff, or in a family with recent hospital contacts, this may not be so.

\section{Specific Infections}

Differentiation between pyogenic and specific infections is usually easy on clinical grounds, provided the latter are kept in mind. In some cases bacteriological confirmation may be difficult. Tuberculosis produces a variety of lesions in the hand-dactylitis, soft tissue abscess, and chronic ulceration at the surface (Fig. I) being the more

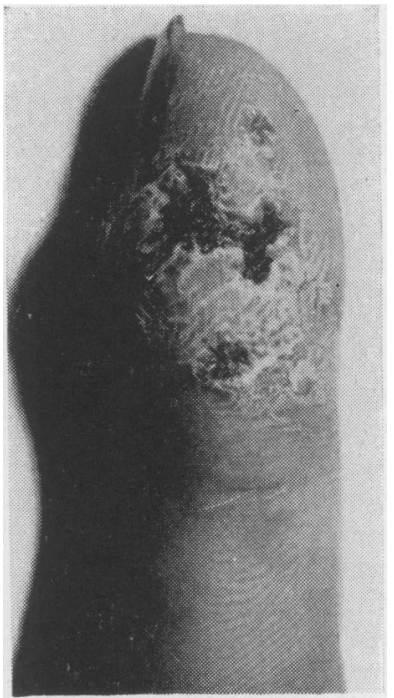

FIG. I.-Tuberculous ulcer of finger-tip.

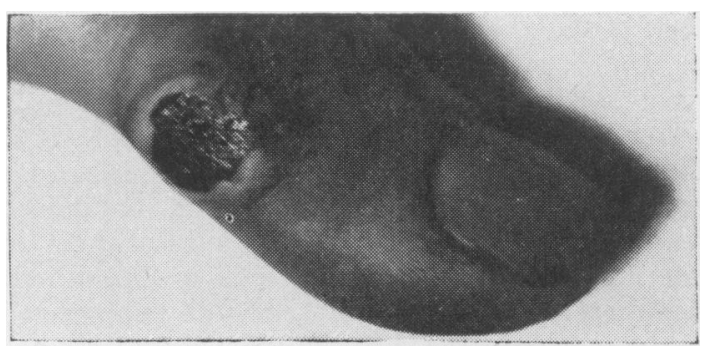

Fig. 2.-Orf.

common. Breakdown in axillary glands occurs more often in the two latter than in bony disease. Orf (Fig. 2) is a virus infection giving rise to a shallow ulcer with a natural limit of about six weeks. Isolation of the virus is difficult but the lesion is commonly secondarily invaded by pyogenic organisms. Erysipeloid is an intradermal inflammation of slow progress, again self-limiting. Confirmation is not possible because there is no discharge and no material available. Biopsy is not justified, because it recovers spontaneously. Diphtheritic infection is secondary to long-standing discharging lesions. It is rare in this country, but 


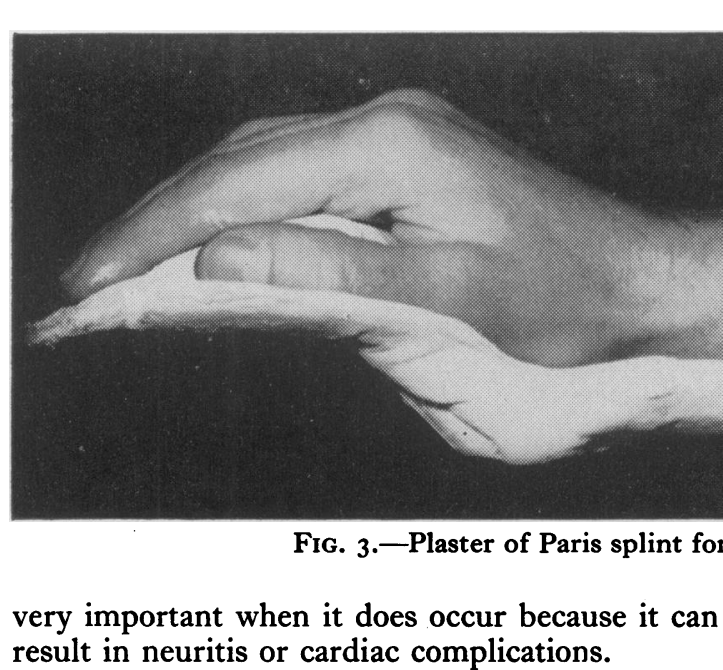

\section{Conservative Treatment}

The management of early cases calls for repeated attendances and daily assessment. It is at this stage that the right decision makes the difference between a short disability with no operation and a long disability, one or more operations, and perhaps permanent disablement at the end of it. Even a small degree of contracture, or a small area of impaired sensation, which could have been avoided, is too high a price to pay in the hand-and residual limitations after septic hands, even now, are not always small.

In hospital clinics about $80 \%$ of septic hand cases attend for the first time with signs of pus. Half the remainder show these signs within a day or two of attendance, and only the remaining $10 \%$ resolve without them. In the community as a whole such figures are quite inapplicable. It is difficult to estimate how often minor hand infections resolve with conservative treatment, or indeed with no treatment at all, because most of them do not attend clinics where records are kept. If treatment is prompt, response should be expected in the majority.

The mainstay of early treatment is rest to the whole hand, and maintenance of adequate blood levels of penicillin. Rest is provided by a comfortable splint to the hand and forearm (Fig. 3), and a large arm sling. The patient is told to put a bolster alongside himself at night to prevent the arm falling outwards and becoming cramped. Penicillin is usually effective, and daily injections of long-acting preparations are adequate.

The first sign of response is an improvement in comfort, and if it is prompt, treatment is continued. Failure to respond to these two measures within 48 hours of beginning treatment is, I believe, presumptive evidence of the presence of pus. It is not presumptive evidence of infection by anti- biotic-resistant organisms, except possibly in the few types of hospital infection already mentioned. It is wrong to wait for 'fluctuation' at the site of infection. It is an unreliable sign in hands, and i it is due to pus it often means extensive involve-iv ment of skin and is followed by an ulcer with delayed healing. Decisions should have been made? earlier than this.

The appearance of pus under the cuticle, or an area under the swelling where tenderness is more marked than elsewhere, or a discharge, are clearco indications to consider surgery.

A few cases need admission to hospital, usuat $\mathrm{y}_{0}$ on general grounds rather than because of theos severity of the infection itself. Sometimes operations last longer than would be advisable ona an out-patient, and medical conditions (such aso diabetes, conditions needing steroid therapy hypertension, and extreme old age) may indicate aOे period of stabilization before operation, or special care afterwards.

\section{Surgical Treatment}

The use of antibiotics before operation is acknowledged to be one of the main factors in 3 . improvement of end-results, as well as one of thei main reasons why many cases resolve without? surgery. It is not established that routine anti-o biotics after operations are of benefit, and there is some evidence that they are not (Anderson, 1958): Choice of antibiotics may vary according to local conditions, but many clinics report, as late as $1962 \%$ (Parsons, 1962), that penicillin is still in general use. $N$

It is not possible to indicate here all the surgicaliv details of approach to abscess cavities in the hand, ${ }^{\omega}$ but a tendency is detected to abandon the routine 'classical' incisions as recommended by Kanavel? (1939) in 1928. A direct approach through moreos limited incisions is more often used. Exploration ${ }_{0}$ of the cavity and cleaning of its ramifications are ${ }_{0}^{\circ}$ followed by closure of the residual space by bandaging. This results in fewer post-operative 


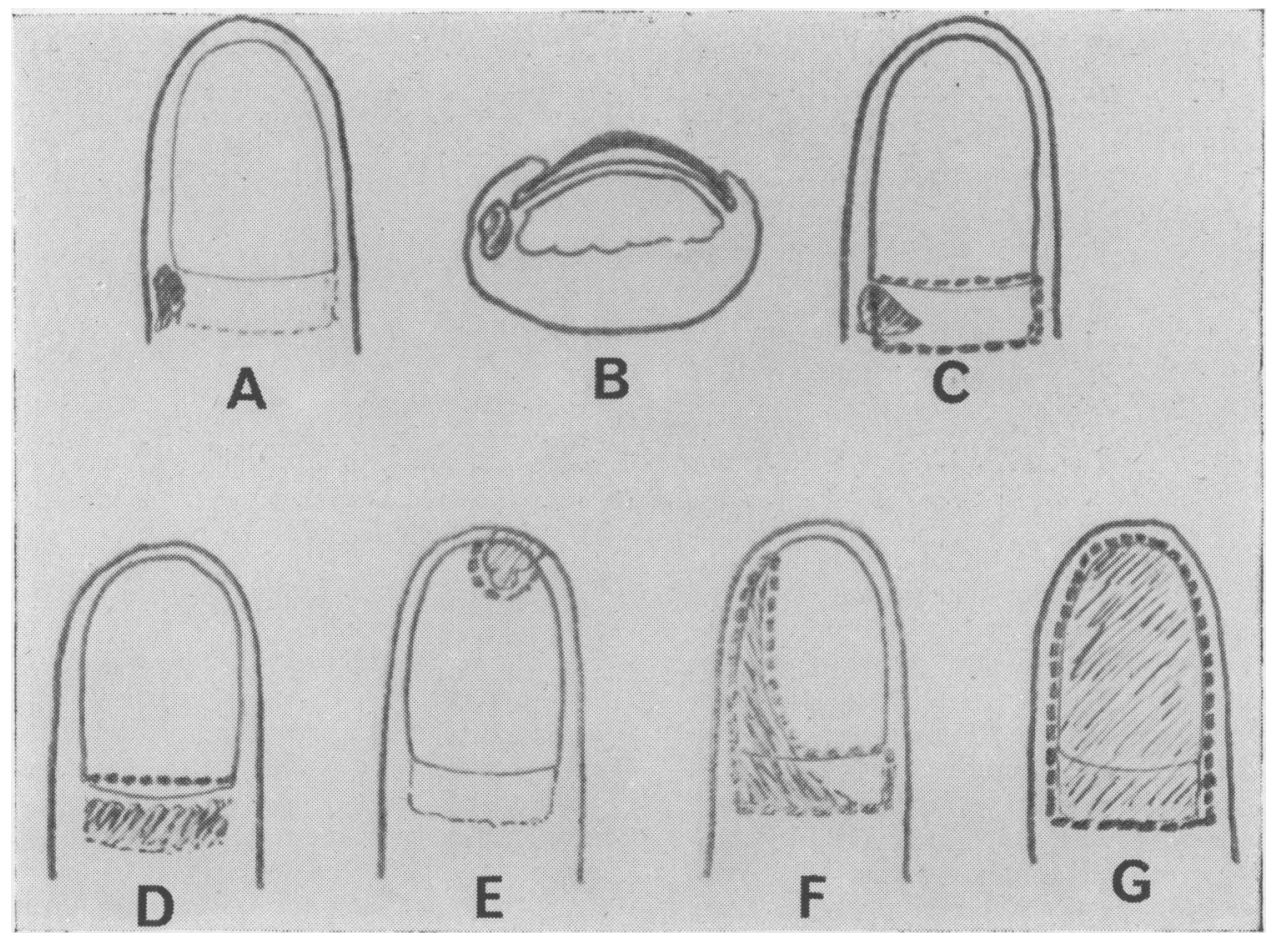

Fig. 4.-Nail removal in treatment of paronychia. $(a)$ and $(b)$ Subcutaneous abscess in sulcus; no nail is removed. (c) Sulcus abscess extending under part of the nailbase; all the nailbase is removed. (d) Extension under all the nailbase; all the nailbase is removed. (e) Distal abscess of nailbed and subcutaneous tissue at the tip; enough nail is removed to expose the involved nailbed fully. $(f)$ Extension under part of nailbase and part of distal nailbed; the whole of nailbase is removed; enough nail is removed distally to expose the involved nailbed fully. $(g)$ Spread under the whole of the nail; all the nail is removed.

dressings, and a better prospect of healing without secondary infection. Drainage by foreign material and counter-incisions are also less frequently needed. Relapse after operation is usually due to a failure to detect the full extent of the lesion, but occasionally to later extension of tissue necrosis or development of osteitis (these two usually in the pulp space) or to an unsuspected foreign body (usually in palm or thenar or hypothenar eminences).

Small incisions are sited where the cavity most nearly approaches the surface, where there is a small sinus, or an area of increased redness and softness of the skin, or a point of maximum tenderness on blunt pressure by the end of a probe. The direction of the incision is determined by the natural creases rather than by the suspected shape of the abscess cavity. An area of skin necrosis already established is often quite adequate for full exploration, without any incision. All cases are operated on under a tourniquet.

The spread of infection under the epidermis is seldom of importance if the blister is trimmed back exactly to its limits and the pus is dried away.
But it sometimes masks the extent of the lesion underneath so that a full assessment of the situation is not made until after the operation has started. It expects of the surgeon a willingness to alter his decisions or even to come to them for the first time while the operation is going on, and the realization that the best results come from suiting the procedure to the particular situation. Each case must be treated on its own merits.

After operation some hands profit by further rest and elevation, but most are comfortable within twenty-four hours. Early function is encouraged. The wound area is kept dry and porous bandages are bette1 than occlusive dressings. The patient is told to keep his whole dressing dry. If he does this it does not need to be changed more often than twice a week.

\section{Paronychia}

This versatile approach to the surgery of the septic hand is illustrated by infections near the nailbed. They may be in the subcutaneous tissue at the base or sulcus, or subcuticularly in the skin of the same region or under the nail in the nailbed 


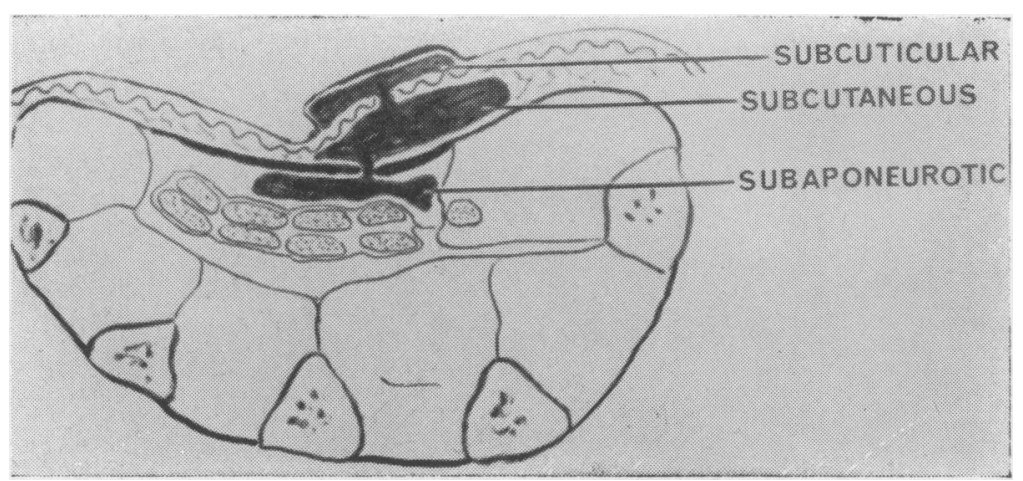

Fig. 5.-The usual situation of deep palmar abscess.

itself. They may be both. The extent of spread in these situations determines the mode of exposure, and the amount of nail, if any, which should be removed. Removing too much nail can leave a raw nailbed, secondarily infected, whose recovery may take considerably longer than the rest of the lesion. Removing too little may cause relapse. Between a quarter and a third of the nail is covered by nailfold, and if pus spreads under this part it has extra difficulty in draining. The whole of the nailbase should be removed (Bailey, I963) so that the fold falls back firmly over its whole extent, and the space under it should be cleaned of debris and macerated cuticle before it is allowed to do so. Extent of spread under the distal part varies from case to case and the amount of nail removal varies correspondingly (Fig. 4).

\section{Pulp Space}

A direct approach to abscesses in the pulp space and removal of all infected material at the primary operation, have reduced the relapse and complication rate. Late, neglected cases are sometimes followed by sequestration of the distal two-thirds of the phalanx; by destruction of the flexor insertion; by invasion of the distal interphalangeal joint-or, most commonly, by development of a thin rigid shell of skin and fibro-fatty tissue, held open by the phalanx in its depths, and creating an uncollapsible cavity, persistently discharging and reinfecting itself. Necrosis of skin and fibro-fatty tissue is responsible for more disability and delay in healing than is any other complication. Delay in recourse to surgery is its cause.

\section{Volar Crease and Volar Compartment}

Some cases, especially in the volar creases, are complicated by local extension into the flexor sheath. It tends to wall itself off without extending into the other parts of the sheath. Generalized swelling of the finger and resistance to passive extension of all joints are therefore unusual.
Limitation of movement at the nearest joint, however, is common. Skin and fibro-fatty destruction, combined with localized adhesion of tendon to sheath and scar, may result in permanent contracture at this joint, and although the clinical course of these infections is less dramatic than in a generalized tendon sheath infection, important limitation of movement may be a permanent aftereffect.

Removal of sloughing skin and fibrous tissue should be done very carefully in these cases, for fear of damaging the sheath before it is knowb whether it is involved or not. Local involvement of the sheath will drain satisfactorily through the local lesion. (See also tendon sheath infection.) ?

\section{Deep Palmar Infection. Subaponeurotic Thenar and Hypothenar Eminences}

Infection and abscess formation extending deep to the aponeurosis, spreading amongst (but rarely into) the tendon sheaths and neurovascular bundles, may be encountered (Fig 5). The subcutaneous cavity is explored, and the exploration is then extended downwards by separating the aponeurosis in the direction of its fibres, and excising a portion of it if there is necrosis of its tissue. It may be necessary to increase exposure by incision, but such extensions can often be sutured at the end of the operation. Although these abscesses cause swelling on the dorsum, there is seldom any indication to provide deeper drainage than that described. Mid-palmar space and thenar space infections, as defined by Kanavel (1939) are very rare occurrences in this country at the present time. They arose from rupture of long-neglected tendon sheath infections, and under modern conditions tendon sheath infections do not progress untreated to such extremes.

\section{Median Nerve Involvement}

Subaponeurotic infections may extend distally under the transverse carpal ligament, and this 
imperils the median nerve. Pressure on it leads to paræsthesia, and perhaps to paresis, if the complication is not borne in mind. Decompression of the carpal tunnel (Bailey, 1963) reverses the process, provided intervention is not delayed. Antibiotic therapy is continued for longer than usual. Suitable support by plaster of Paris splint and careful bandaging prevent prolapse of the flexor tendons. Physiotherapy is often needed.

\section{Tendon Sheath}

Rapid spread through the whole of the flexor sheath, usually after a small penetrating wound on the volar surface, produces a painful, swollen finger, with maximum tenderness at the proximal end of the sheath-or, in the case of the ulnar bursa, at the level of the middle of the fifth metacarpal bone. These infections are usually streptococcal, and early treatment with penicillin, and local rest, is followed by complete resolution in the majority. Failure to resolve promptly or, perhaps, resolution of pain but persistence of the swelling and limitation of movement (especially limitation of passive extension) indicate the need for surgical intervention. The proximal end of the sheath is exposed by a limited incision in the skin and the sheath decompressed by a small incision into it. The sheath is irrigated with penicillin (10,000 $\mathrm{u}$. per $\mathrm{ml}$.) through a ureteric catheter before closure of the skin wound. Further antibiotic therapy, together with splintage, until resolution is complete, are then indicated. Physiotherapy is occasionally needed to encourage full return to function. The majority of results in this condition are now good, provided the case presents for treatment within the first forty-eight hours. The severe pain of the early stages usually ensures that it does.

Local infection of the sheath from established staphylococcal infection in the volar subcutaneous spaces (q.v.) is treated quite differently. Nevertheless, some cases are seen in which local suppuration, well contained, is associated with a sympathetic effusion into the more proximal part of the sheath. These cases produce a confusing clinical picture. The safest approach to them is firstly to treat the local (and usually distal) tendon involvement by cleaning out the subcutaneous infection. This allows any sheath abscess to drain through this area. The effusion into the proximal section of the sheath is then approached at its proximal end by a separate operation. It is decompressed, as described above, without breaking through to the distal abscess. If the barrier between the two parts of the sheath is broken down, it may result in rapid extension of suppuration proximally and ultimate loss of function of the whole tendon.

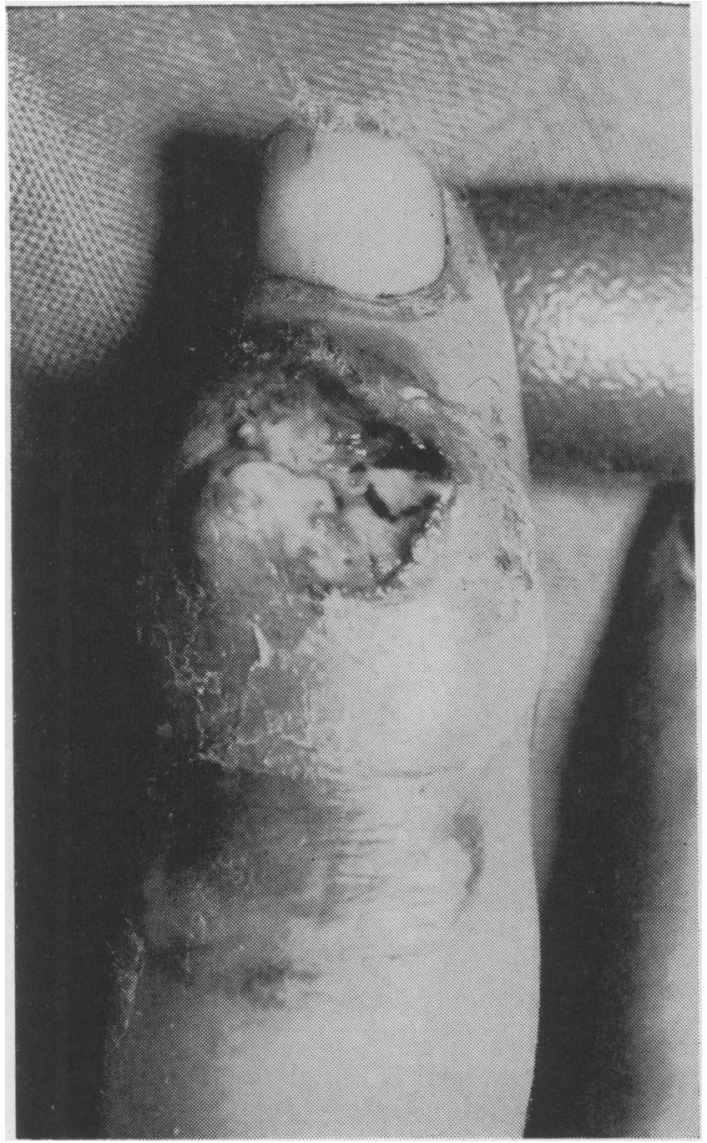

FIG. 6.-Acute suppurative arthritis in a chronic arthritic joint.

\section{Suppurative Arthritis}

Suppuration in interphalangeal joints occurs after penetrating wounds, or by spread from adjacent subcutaneous infections or-rarelyafter infection of the tendon sheath. It takes a long time to heal. Chronic arthritic joints in the elderly are very susceptible (Figs. 6 and 7). Exploration of the joint (from the side, or through a damaged extensor tendon) may reduce the healing time if all infected material, sequestra, or necrotic cartilage are removed by gentle curettage. It may be possible to suture the wound at the end of the operation (Bailey, 1963). Often all that can be hoped for, especially in the elderly, is an ankylosis in good position, and an early decision to amputate may be correct-particularly where the proximal interphalangeal joint or metacarpophalangeal joint is involved in the younger subject. Where suppurative arthritis is associated with extensive soft tissue necrosis, and sometimes tendon necrosis also, early amputation becomes even more appropriate (Fig. 8). 


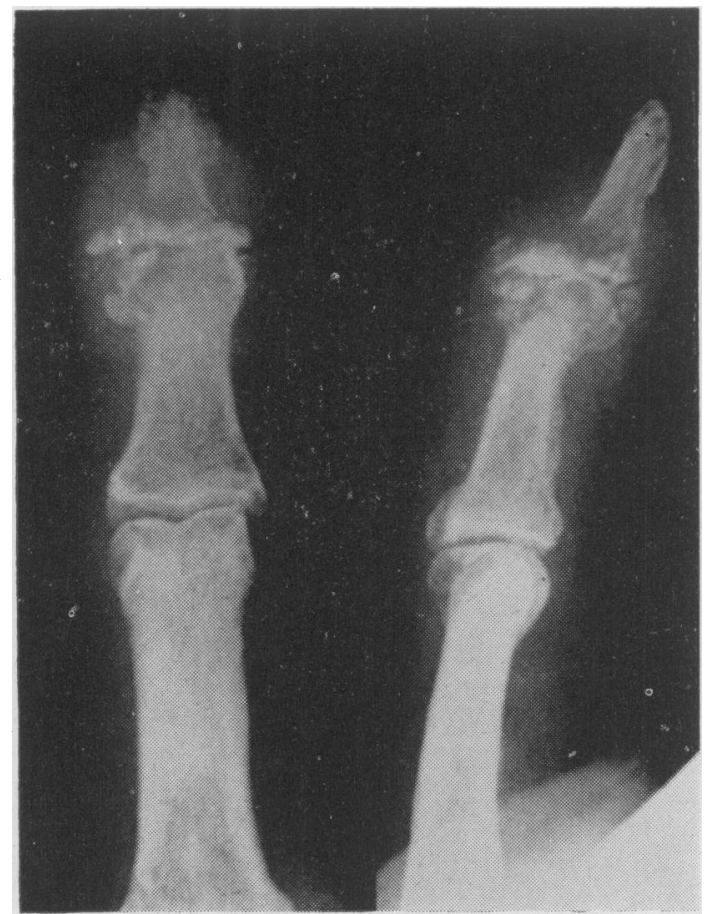

FIG. 7.-X-ray appearance of case shown in Fig. 6.

\section{Secondary Operations}

Surgical treatment is not always complete when the infection is overcome. Permanent disability is the result of scarring, and early attempts to minimize scar formation are an important part of the management of septic hands. Skin replacement, as soon as the infected area will accept it, is the most effective procedure. Wherever spontaneous closure cannot be anticipated within a few days, grafting should be considered. Delay in decision commits the surgeon to an operation on reinfected granulations with a thickened fibrous base, in which the tendency to contract is already established. It is not possible to elaborate this subject here, because different cases pose different problems, to be countered by different details.

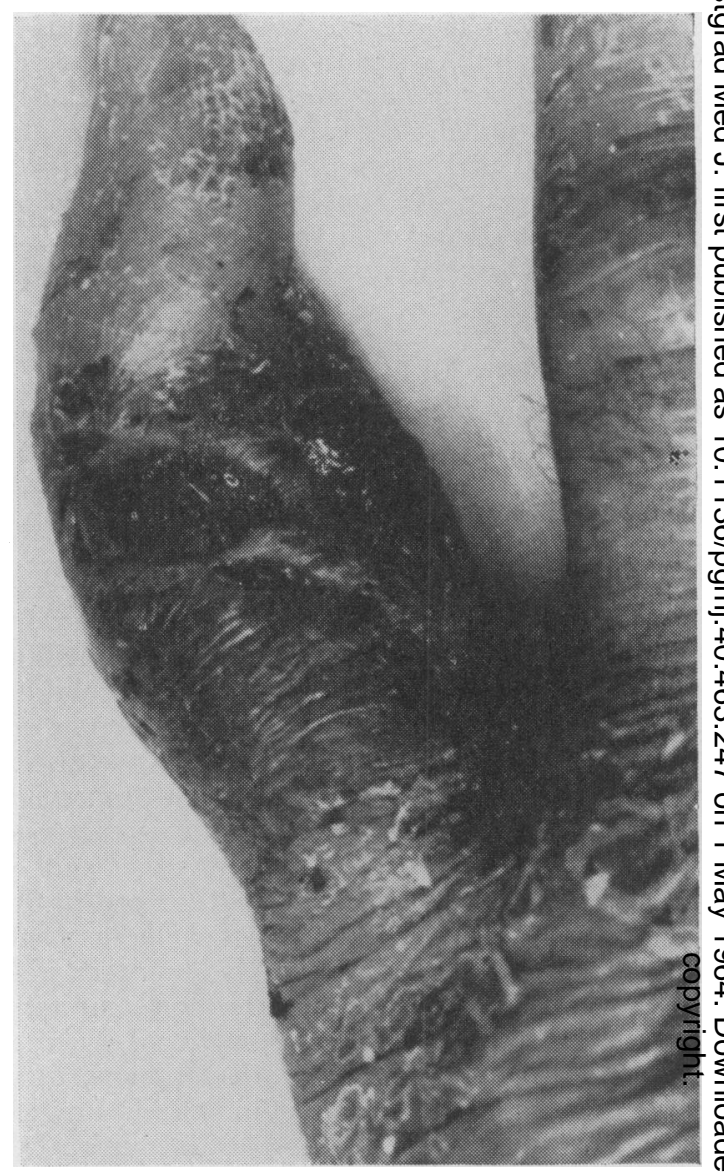

FIG. 8.-Suppurative arthritis, osteitis of phalanx, and suppurative tenosynovitis in one finger-a case for $\overline{\overrightarrow{0}}$ amputation.

But it is important to emphasize that the modern? treatment of septic hands involves not only을 consideration of the infecting agent and of over- - . coming it, but of vigorous and early attempts to repair the damage as completely as possible.

Figs. 1, 2, 3, 5, 6, 7 and 8 are reproduced from 'The Casualty Department' by kind permission of E. \& S.을 Livingstone \& Co., Edinburgh and London.

\section{REFERENCES}

Anderson, J. (1958): Dispensibility of Post-operative Penicillin in Septic Hand Surgery, Brit. med. F., ii, 1569.

Bailey, David A. (1963): 'The Infected Hand'. London: H. K. Lewis.

Kanavel, A. B. (r939): 'Infections in the Hand'. Philadelphia: Lea and Febiger.

Parsons, D. W. (1962): Treatment of the Septic Hand, Proc. roy. Soc. Med., 55, 445.

Williams, R. E. O., and Miles, A. A. (1949): Infection and Sepsis in Industrial Wounds of the Hand. Spec. Rep. Serco med. Res. Coun. (Lond.), No. 266. 